\title{
HIGH-RESOLUTION LIDAR DATA FOR THE CHILKAT RIDGE AREA, ALASKA
}

Ronald P. Daanen and Katreen Wikstrom Jones

Raw Data File 2019-7

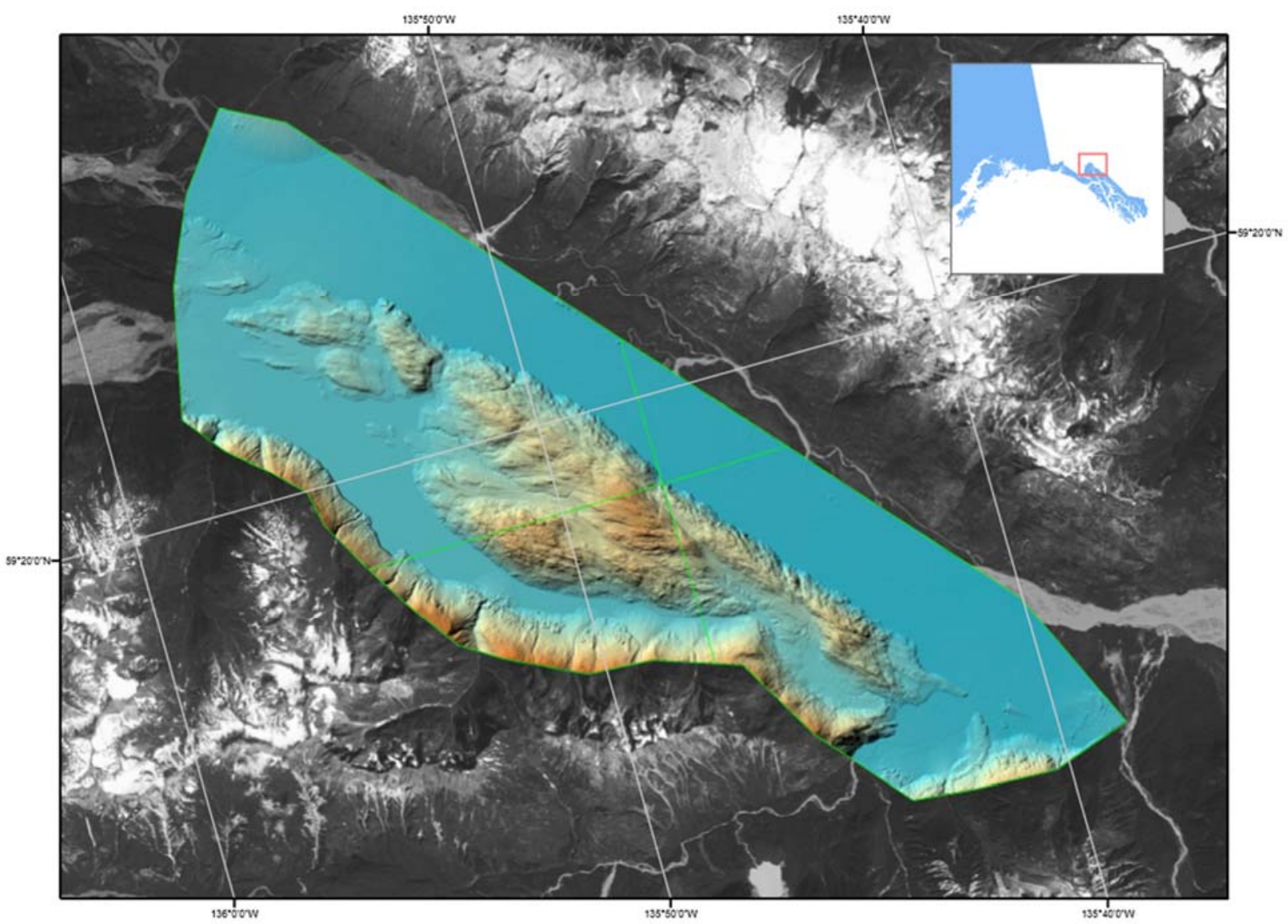

This report has not been reviewed for technical content or for conformity to the editorial standards of DGGS.

2020

STATE OF ALASKA

DEPARTMENT OF NATURAL RESOURCES

DIVISION OF GEOLOGICAL \& GEOPHYSICAL SURVEYS
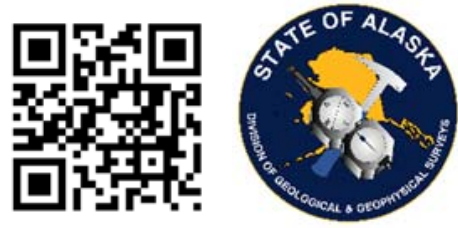
STATE OF ALASKA

Mike Dunleavy, Governor

DEPARTMENT OF NATURAL RESOURCES

Corri A. Feige, Commissioner

\section{DIVISION OF GEOLOGICAL \& GEOPHYSICAL SURVEYS}

Steve Masterman, State Geologist \& Director

Publications produced by the Division of Geological \& Geophysical Surveys are available to download from the DGGS website (dggs.alaska.gov). Publications on hard-copy or digital media can be examined or purchased in the Fairbanks office:

\section{Alaska Division of Geological \& Geophysical Surveys (DGGS)}

3354 College Road | Fairbanks, Alaska 99709-3707

Phone: 907.451.5010 | Fax 907.451.5050

dggspubs@alaska.gov | dggs.alaska.gov

DGGS publications are also available at:

Alaska State Library, Historical

Collections \& Talking Book Center

395 Whittier Street

Juneau, Alaska 99801

Alaska Resource Library and

Information Services (ARLIS)

3150 C Street, Suite 100

Anchorage, Alaska 99503

\section{Suggested citation:}

Daanen, R.D., and Wikstrom Jones, Katreen, 2020, High-resolution lidar data for the Chilkat Ridge area, Alaska: Alaska Division of Geological \& Geophysical Surveys Raw Data File 2019-7, 11 p. http://doi.org/10.14509/30224
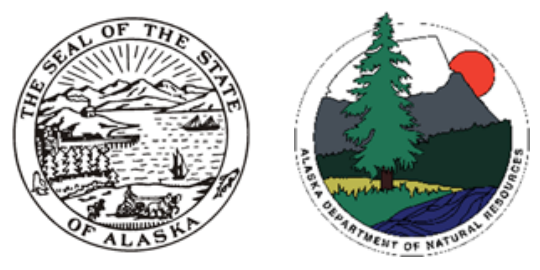


\section{HIGH-RESOLUTION LIDAR DATA FOR THE CHILKAT RIDGE AREA, ALASKA}

Ronald P. Daanen ${ }^{1}$ and Katreen Wikstrom Jones ${ }^{1}$

\section{ABSTRACT}

The State of Alaska Division of Geological \& Geophysical Surveys (DGGS) used lidar to produce a digital terrain model (DTM) and digital surface model (DSM) over ridgelines along the Chilkat River just north of Haines, Alaska. The lidar and Global Navigation Satellite System (GNSS) data were collected on November 15-16, 2018, and processed using Terrasolid. This data collection is being released as a Raw Data File with an open end-user license. All files can be downloaded free of charge from the DGGS website.

\section{INTRODUCTION}

This dataset includes point cloud data, a 32-bit digital terrain model, and an intensity image covering a study area near the Chilkat Ridge region of southeast Alaska. The No Data value is set to $-3.40282306074 \mathrm{e}+038$. The data release is one of a series of Alaska Division of Geological \& Geophysical Surveys (DGGS) publications to present elevation data. The goal is to provide information for the Alaska Division of Forestry on the ground conditions and tree cover on Chilkat Ridge.

\section{LIST OF DELIVERABLES}

Classified Points

DSM and DTM

Intensity

Metadata

\section{MISSION PLAN}

\section{Aircraft and Instrument}

DGGS operates a Riegle VUX1-LR lidar integrated with a GNSS and Northrop Grumman IMU system. The integration was designed by Phoenix Lidar Systems. This survey was flown on a fixed-wing aircraft configuration, using a Cessna 185 operated by Clearwater Air, Inc., of Anchorage, Alaska. Ground control points were collected using a Trimble Navigation Limited GPS consisting of a Trimble R7 base station and R8 rover system.

\footnotetext{
${ }^{1}$ Alaska Division of Geological \& Geophysical Surveys, 3354 College Road, Fairbanks, Alaska 99709
} 


\section{Weather Conditions and Flight Times}

The lidar data were collected on November 15, 2018, and November 16, 2018. Most of Chilkat Ridge was collected on the first day of the survey, which started at 10:48 AM and ended at 3:58 PM. The sky was mostly clear, with a few persistent low-level fog banks and a temperature just above freezing. The second day of flying was also clear, with less low-hanging fog and temperatures just above freezing. Takeoff for that day was at 8:41 AM and landing was at 11:57 AM. No abnormalities were observed during the flights.

\section{PROCESSING REPORT}

\section{Lidar Dataset Processing}

Due to dense vegetation and the need to provide a bare earth elevation model with a resolution of $1 \mathrm{~m}$, we designed the survey to collect approximately 40 points per square meter. This resulted in an average of about 3 points per square meter on the ground. We also designed the survey to have significant overlap between scan lines to capture all gaps in the vegetation.

Raw data were processed using Terrasolid software to produce integrated files for navigation correction and a point cloud for calibration. The navigation was corrected using Inertial Explorer software, where the GNNS and IMU data are integrated to establish correct flight path and orientation of the lidar sensor. The data were processed multiple times, resulting in position accuracies illustrated in figure 1.

Internal lidar point cloud data were calibrated using Terrasolid software. The initial accuracy of the point cloud was $8.346 \mathrm{~cm}$. After calibration, the point cloud had an average magnitude accuracy of $6.914 \mathrm{~cm}$.

The point cloud is classified for ground points as well as low, medium, and high vegetation (0.01-0.3 $\mathrm{m}, 0.3-5 \mathrm{~m}$, and 5-60 $\mathrm{m}$ heights above the ground, respectively). Some manual processing was required to eliminate fog and misclassified ground points. All low points and air points are eliminated from the dataset. Lastly, the DSM and DTM were hydroflattened to mean surrounding elevation for all lakes and ponds. 


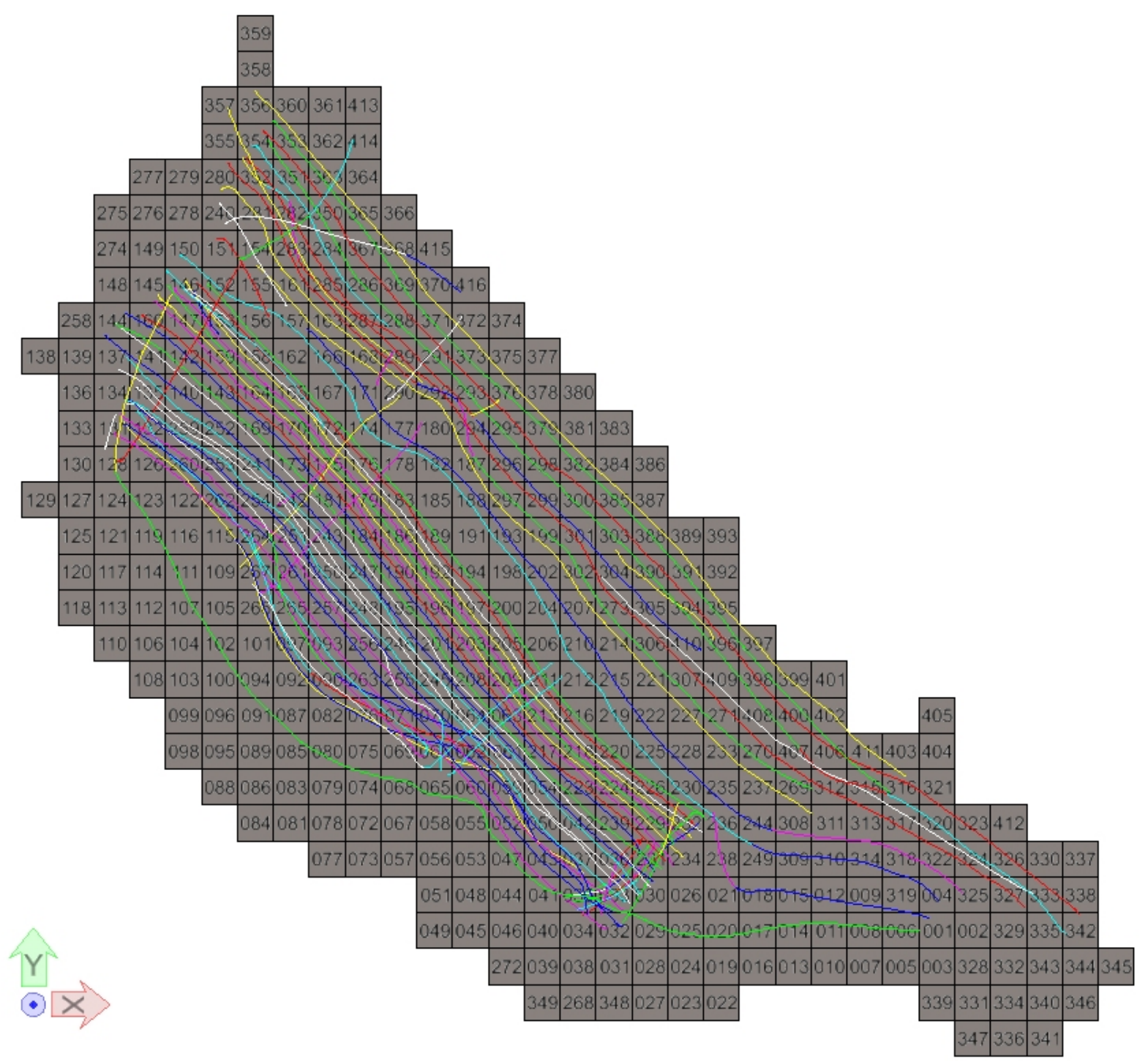

Figure 1. Flight lines included in the point cloud data. The lidar swath width averages 750 meters wide centered on the flight line but is dependent on local terrain. The point cloud tiles, labeled with las file numbers, are $1 \mathrm{~km}$ wide.

\section{DTM}

The ground points from the final point cloud were used to build the digital terrain model in ArcGIS. The point cloud was loaded as a las dataset and filtered for ground points. The remaining points were used in a las dataset-to-raster conversion tool. Rasters, with a ground pixel resolution of 1 meter, were derived from mean values from a 2-meter sampling distance.

\section{DSM}

The digital surface model was created from the first returns in the point cloud. Due to a large number of points in vegetation, we used a binning method with natural neighbor gap-filling. 
The $1 \mathrm{~m}$ bins did not gap-fill correctly in the entire dataset, however, so we opted to store the DSM in a $2-\mathrm{m}$-resolution raster.

\section{Intensity}

The intensity raster is provided using the ground points only. The raster resolution is $1 \mathrm{~m}$.

\section{SURVEY REPORT}

\section{Ground Control}

Surveyed ground control, captured with a Trimble RTK GPS, provided both control points for calibration and checkpoints for accuracy assessment of the lidar point cloud. A snow crust was observed on most ground surfaces but was less than $5 \mathrm{~cm}$ thick and highly variable. This crust of snow is not expected to affect the elevation map beyond the overall accuracy capability of the survey. All points collected were in natural terrain with variable amounts of vegetated cover.

Control point adjustment involves a linear transformation of the entire lidar point cloud based on the mean elevation difference between surveyed ground data and an interpolated TIN (triangular irregular network) of the point cloud. The collected control points compared with lidar ground points had an average difference of $-3 \mathrm{~cm}$ that was used to correct the elevation of all points in the point cloud. The quality of the control and checkpoints is summarized in Appendices 1 and 2 .

Geometric accuracy of the point cloud is similarly estimated using a derived TIN in conjunction with surveyed ground data. The final elevation difference of the ground point elevation and the bare earth model, calculated as the average error compared to checkpoints, was $0.5 \mathrm{~cm}$ (Appendix 1). The Root Mean Square Error of the final data layers for the bare earth elevation between the checkpoints and lidar ground points is $8.5 \mathrm{~cm}$.

\section{Coordinate system and Datum}

All data were processed and delivered in UTM NAD83 (2011) and vertical datum NAVD88 with a GEOID correction following the latest GEOID12B for Alaska.

\section{QA/QC REPORT}

The quality of the data is discussed in the previous paragraphs as average elevation compared to an independent dataset. The second aspect of the quality of the data is related to point density distribution over the survey area (fig. 2). The average point density is 40 points per square meter, with ground returns of 3 points per square meter. 


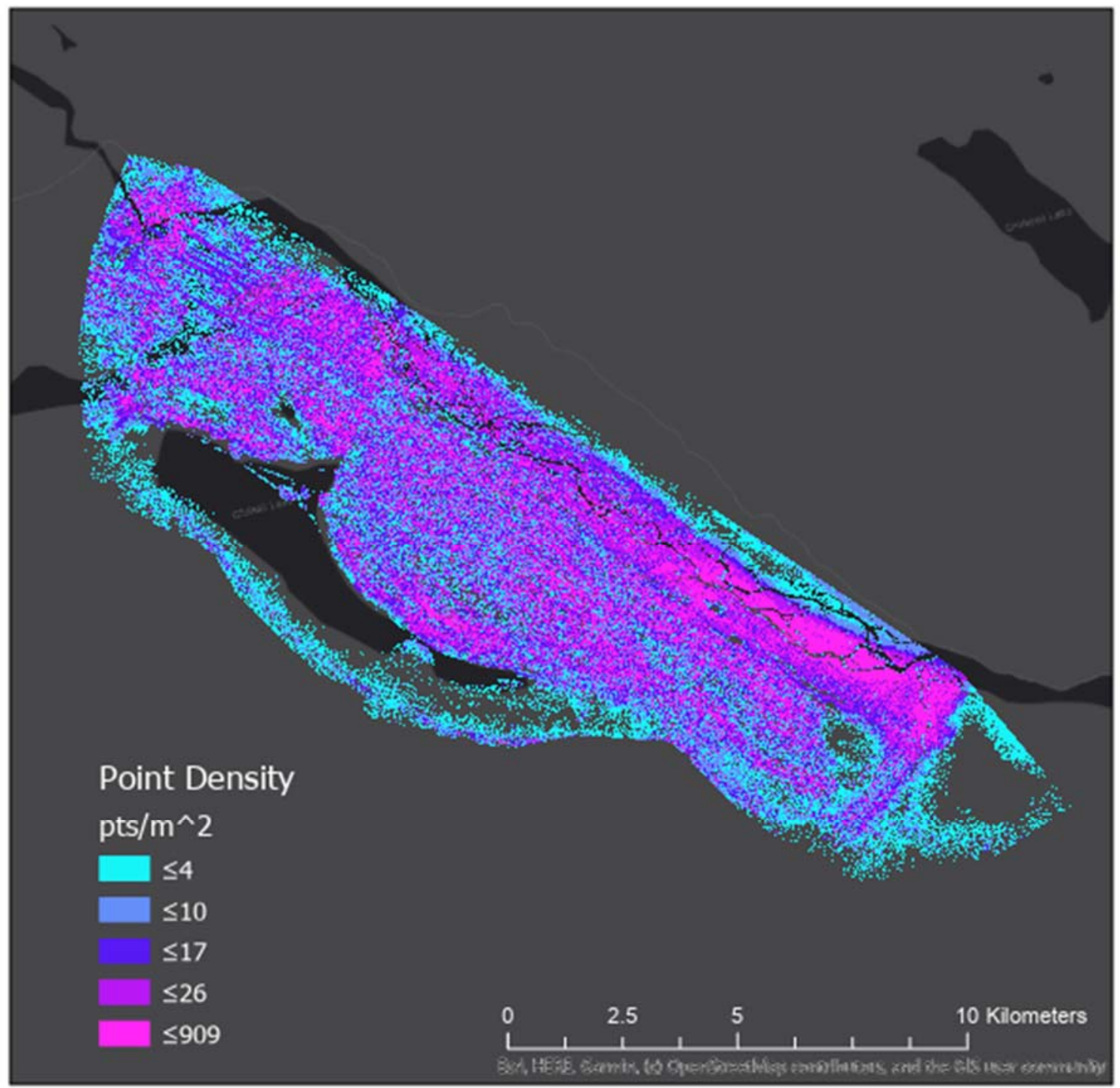

Figure 2. Ground point density displayed as a 1-meter raster for the survey, with 50 percent transparency over the hillshade derived from the DTM; cells without data are shown in white. 
Appendix 1. Checkpoints

\begin{tabular}{|c|c|c|c|c|c|c|}
\hline FID & & $\begin{array}{c}\text { Easting } \\
(\mathrm{m})\end{array}$ & $\begin{array}{l}\text { Northing } \\
\text { (m) }\end{array}$ & $\begin{array}{c}\text { Known Z } \\
\text { (m) }\end{array}$ & $\begin{array}{c}\text { Laser Z } \\
(\mathrm{m})\end{array}$ & $\begin{array}{l}\mathrm{Dz} \\
(\mathrm{m})\end{array}$ \\
\hline gcp48day2 & 1 & 445277.2 & 6581641 & 67.319 & removed & * \\
\hline gcp-52day1 & 2 & 456397.5 & 6578681 & 20.252 & 20.42 & 0.168 \\
\hline gcp130day2 & 3 & 449208.4 & 6584774 & 39.548 & 39.71 & 0.162 \\
\hline gcp91day2 & 4 & 453149 & 6573180 & 60.599 & 60.75 & 0.151 \\
\hline gcp27day2 & 5 & 445532 & 6581742 & 66.287 & 66.43 & 0.143 \\
\hline gcp100day2 & 6 & 450588.1 & 6574668 & 60.304 & 60.43 & 0.126 \\
\hline gcp28day2 & 7 & 445576.5 & 6581745 & 65.741 & 65.86 & 0.119 \\
\hline gсp-53day1 & 8 & 456370.1 & 6578719 & 20.306 & 20.4 & 0.094 \\
\hline gcp132day2 & 9 & 449247.6 & 6584774 & 39.459 & 39.55 & 0.091 \\
\hline gcp-36day1 & 10 & 452578.4 & 6581795 & 34.073 & 34.15 & 0.077 \\
\hline gcp-23day1 & 11 & 467974.2 & 6569095 & 9.452 & 9.52 & 0.068 \\
\hline gсp-47day1 & 12 & 457455.8 & 6577192 & 18.687 & 18.73 & 0.043 \\
\hline gcp-46day1 & 13 & 457826.1 & 6576663 & 17.37 & 17.41 & 0.04 \\
\hline gcp81day2 & 14 & 455067.6 & 6576432 & 19.481 & 19.52 & 0.039 \\
\hline gcp-34day1 & 15 & 452541.7 & 6581926 & 34.633 & 34.67 & 0.037 \\
\hline gcp-22day1 & 16 & 468001.3 & 6569083 & 9.515 & 9.55 & 0.035 \\
\hline gcp29day2 & 17 & 445596.5 & 6581739 & 65.492 & 65.52 & 0.028 \\
\hline gcp11day2 & 18 & 450424.3 & 6579756 & 226.263 & 226.29 & 0.027 \\
\hline gcp-27day1 & 19 & 452457.3 & 6581849 & 33.262 & 33.28 & 0.018 \\
\hline gcp-56day1 & 20 & 456241.2 & 6578905 & 20.407 & 20.42 & 0.013 \\
\hline gcp69day2 & 21 & 451315.3 & 6580492 & 27.937 & 27.93 & -0.007 \\
\hline gcp62day2 & 22 & 449836.6 & 6582579 & 41.02 & 41.01 & -0.01 \\
\hline gcp-20day1 & 23 & 468000.5 & 6569091 & 9.42 & 9.4 & -0.02 \\
\hline gсp149day2 & 24 & 451656 & 6583383 & 34.163 & 34.14 & -0.023 \\
\hline gcp6day2 & 25 & 453974.9 & 6575010 & 535.946 & 535.92 & -0.026 \\
\hline gcp-44day1 & 26 & 457933.2 & 6576547 & 19.617 & 19.59 & -0.027 \\
\hline gcp126day2 & 27 & 448326.3 & 6584968 & 38.8 & 38.77 & -0.03 \\
\hline gcp-24day1 & 28 & 452450.5 & 6581898 & 33.215 & 33.18 & -0.035 \\
\hline gcp58day2 & 29 & 448480.7 & 6583537 & 43.977 & 43.94 & -0.037 \\
\hline gcp60day2 & 30 & 448445.3 & 6583561 & 43.903 & 43.86 & -0.043 \\
\hline gcp63day2 & 31 & 449831.5 & 6582559 & 41.024 & 40.97 & -0.054 \\
\hline gcp33day2 & 32 & 445677.6 & 6581646 & 65.639 & 65.58 & -0.059 \\
\hline gcp147day2 & 33 & 451624.1 & 6583432 & 34.35 & 34.29 & -0.06 \\
\hline gcp148day2 & 34 & 451630.3 & 6583410 & 34.452 & 34.39 & -0.062 \\
\hline gcp-37day1 & 35 & 452633.7 & 6581770 & 34.213 & 34.15 & -0.063 \\
\hline gcp135day2 & 36 & 450934.6 & 6583951 & 35.929 & 35.86 & -0.069 \\
\hline gcp-42day1 & 37 & 457876.9 & 6576624 & 19.474 & 19.4 & -0.074 \\
\hline gcp8day2 & 38 & 453993.3 & 6575011 & 536.019 & 535.94 & -0.079 \\
\hline gcp146day2 & 39 & 451617.3 & 6583423 & 34.339 & 34.25 & -0.089 \\
\hline
\end{tabular}




\begin{tabular}{|c|c|c|c|c|c|c|}
\hline FID & & $\begin{array}{c}\text { Easting } \\
(\mathrm{m})\end{array}$ & $\begin{array}{l}\text { Northing } \\
\text { (m) }\end{array}$ & $\begin{array}{c}\text { Known Z } \\
(\mathrm{m})\end{array}$ & $\begin{array}{l}\text { Laser Z } \\
\text { (m) }\end{array}$ & $\begin{array}{r}\text { Dz } \\
(\mathrm{m})\end{array}$ \\
\hline gсp159day2 & 40 & 452394.3 & 6582459 & 34.221 & 34.13 & -0.091 \\
\hline gcp7day2 & 41 & 453979.3 & 6575020 & 535.882 & 535.79 & -0.092 \\
\hline gcp140day2 & 42 & 450954.4 & 6583961 & 36.606 & 36.51 & -0.096 \\
\hline gcp142day2 & 43 & 450916.6 & 6583995 & 36.821 & 36.7 & -0.121 \\
\hline gcp112day2 & 44 & 448009.6 & 6585968 & 85.432 & 85.29 & -0.142 \\
\hline gcp162day2 & 45 & 452411 & 6582385 & 34.68 & 34.53 & -0.15 \\
\hline gcp114day2 & 46 & 448051.8 & 6585932 & 87.134 & 86.97 & -0.164 \\
\hline gcp122day2 & 47 & 448218.1 & 6585827 & 92.734 & removed & * \\
\hline gcp106day2 & 48 & 447909.9 & 6586079 & 80.231 & removed & * \\
\hline gсp-10day1 & 49 & 469261 & 6567800 & 9.399 & outside & * \\
\hline gcp161day2 & 50 & 452405.4 & 6582400 & 34.412 & outside & * \\
\hline gcp20day2 & 51 & 445353.2 & 6581608 & 67.808 & outside & * \\
\hline gcp22day2 & 52 & 445405.2 & 6581613 & 67.605 & outside & * \\
\hline gcp25day2 & 53 & 445460.6 & 6581673 & 67.012 & outside & * \\
\hline gcp26day2 & 54 & 445485.9 & 6581701 & 66.725 & outside & * \\
\hline gcp37day2 & 55 & 445516.3 & 6581502 & 66.497 & outside & * \\
\hline gcp42day2 & 56 & 445231.6 & 6581552 & 68.48 & outside & * \\
\hline gcp47day2 & 57 & 445269.8 & 6581658 & 68.386 & outside & * \\
\hline gcp49day2 & 58 & 445288.1 & 6581624 & 68.192 & outside & * \\
\hline gcp76day2 & 59 & 453713.8 & 6578205 & 22.312 & outside & * \\
\hline hain319sday1 & 60 & 469531.4 & 6567800 & 11.418 & outside & * \\
\hline Average dz & -0.005 & & & & & \\
\hline Minimum dz & -0.164 & & & & & \\
\hline Maximum dz & 0.168 & & & & & \\
\hline $\begin{array}{c}\text { Average } \\
\text { magnitude of } \\
\text { deviation }\end{array}$ & 0.071 & & & & & \\
\hline $\begin{array}{c}\text { Root mean } \\
\text { square }\end{array}$ & 0.085 & & & & & \\
\hline $\begin{array}{c}\text { Standard } \\
\text { deviation }(n=45)\end{array}$ & 0.086 & & & & & \\
\hline
\end{tabular}


Appendix 2. Control Points

\begin{tabular}{|c|c|c|c|c|c|c|}
\hline FID & & $\begin{array}{l}\text { Easting } \\
\text { (m) }\end{array}$ & $\begin{array}{l}\text { Northing } \\
(\mathrm{m})\end{array}$ & $\begin{array}{c}\text { Known Z } \\
(\mathrm{m})\end{array}$ & $\begin{array}{c}\text { Laser Z } \\
\text { (m) }\end{array}$ & $\mathrm{DZ}(\mathrm{m})$ \\
\hline - & & 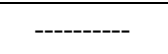 & ---------- & ------ & ------- & ------- \\
\hline gср38day2 & 1 & 445484.3 & 6581506 & 66.261 & removed & $*$ \\
\hline gcp17day2 & 2 & 447647.2 & 6580958 & 75.708 & removed & $*$ \\
\hline gcp13day2 & 3 & 447642.4 & 6580935 & 75.654 & removed & $*$ \\
\hline gcp14day2 & 4 & 447630.6 & 6580946 & 75.772 & removed & $*$ \\
\hline gcp90day2 & 5 & 453147.3 & 6573189 & 60.652 & 60.83 & 0.178 \\
\hline gcp92day2 & 6 & 453148.9 & 6573175 & 60.534 & 60.7 & 0.166 \\
\hline gcp155day2 & 7 & 452430.7 & 6582380 & 35.135 & 35.3 & 0.165 \\
\hline gcp89day2 & 8 & 459224.1 & 6570722 & 13.72 & 13.88 & 0.16 \\
\hline gcp86day2 & 9 & 459214.3 & 6570707 & 13.758 & 13.91 & 0.152 \\
\hline gcp87day2 & 10 & 459207.9 & 6570714 & 13.76 & 13.91 & 0.15 \\
\hline gcp-55day1 & 11 & 456310.2 & 6578802 & 20.303 & 20.45 & 0.147 \\
\hline gcp5day2 & 12 & 454881.6 & 6574193 & 447.094 & 447.24 & 0.146 \\
\hline gcp16day2 & 13 & 447639.9 & 6580958 & 75.786 & 75.93 & 0.144 \\
\hline gcp72day2 & 14 & 452557.5 & 6578937 & 23.951 & 24.09 & 0.139 \\
\hline gcp88day2 & 15 & 459218.2 & 6570722 & 13.817 & 13.95 & 0.133 \\
\hline gcp84day2 & 16 & 456967.7 & 6573558 & 15.138 & 15.27 & 0.132 \\
\hline gcp15day2 & 17 & 447630.4 & 6580957 & 75.831 & 75.96 & 0.129 \\
\hline gcp133day2 & 18 & 449271.9 & 6584776 & 38.842 & 38.96 & 0.118 \\
\hline gcp-54day1 & 19 & 456343.2 & 6578757 & 20.236 & 20.35 & 0.114 \\
\hline gcp93day2 & 20 & 453142.2 & 6573178 & 60.535 & 60.64 & 0.105 \\
\hline gcp3day2 & 21 & 454878.9 & 6574210 & 447.188 & 447.29 & 0.102 \\
\hline gcp70day2 & 22 & 452565.7 & 6578952 & 24.011 & 24.11 & 0.099 \\
\hline gcp2day2 & 23 & 454865.1 & 6574213 & 447.195 & 447.29 & 0.095 \\
\hline gcp83day2 & 24 & 456974.4 & 6573562 & 15.148 & 15.24 & 0.092 \\
\hline gcp-58day1 & 25 & 456145.1 & 6579038 & 20.649 & 20.74 & 0.091 \\
\hline gcp131day2 & 26 & 449225.6 & 6584774 & 39.63 & 39.72 & 0.09 \\
\hline gcp94day2 & 27 & 453142.3 & 6573178 & 60.552 & 60.64 & 0.088 \\
\hline gcp97day2 & 28 & 450571.4 & 6574655 & 60.849 & 60.93 & 0.081 \\
\hline gcp-29day1 & 29 & 452461.8 & 6581635 & 28.189 & 28.27 & 0.081 \\
\hline gcp-61day1 & 30 & 454642.6 & 6580691 & 27.859 & 27.94 & 0.081 \\
\hline gcp71day2 & 31 & 452568.3 & 6578942 & 23.959 & 24.04 & 0.081 \\
\hline gcp-19day1 & 32 & 467978.6 & 6569101 & 9.41 & 9.49 & 0.08 \\
\hline gcp85day2 & 33 & 456964.6 & 6573560 & 15.14 & 15.22 & 0.08 \\
\hline gcp-31day1 & 34 & 452497.1 & 6581572 & 28.29 & 28.36 & 0.07 \\
\hline gcp-25day1 & 35 & 452447 & 6581882 & 33.221 & 33.29 & 0.069 \\
\hline gcp-57day1 & 36 & 456198.2 & 6578965 & 20.442 & 20.51 & 0.068 \\
\hline gcp-28day1 & 37 & 452451 & 6581690 & 28.404 & 28.47 & 0.066 \\
\hline gcp-30day1 & 38 & 452485.8 & 6581591 & 28.337 & 28.4 & 0.063 \\
\hline gcp10day2 & 39 & 450423.6 & 6579770 & 226.066 & 226.12 & 0.054 \\
\hline
\end{tabular}




\begin{tabular}{|c|c|c|c|c|c|c|}
\hline FID & & $\begin{array}{c}\text { Easting } \\
(\mathrm{m})\end{array}$ & $\begin{array}{l}\text { Northing } \\
(\mathrm{m})\end{array}$ & $\begin{array}{c}\text { Known Z } \\
(\mathrm{m})\end{array}$ & $\begin{array}{c}\text { Laser Z } \\
(\mathbf{m})\end{array}$ & $\mathrm{DZ}(\mathrm{m})$ \\
\hline gcp134day2 & 40 & 449308.7 & 6584778 & 38.206 & 38.26 & 0.054 \\
\hline gcp73day2 & 41 & 453721 & 6578233 & 21.958 & 22.01 & 0.052 \\
\hline gcp-48day1 & 42 & 457459.8 & 6577159 & 18.642 & 18.69 & 0.048 \\
\hline gcp-60day1 & 43 & 454762.1 & 6580570 & 26.683 & 26.73 & 0.047 \\
\hline gcp-32day1 & 44 & 452508.5 & 6581615 & 28.256 & 28.3 & 0.044 \\
\hline gcp78day2 & 45 & 455094.8 & 6576448 & 19.599 & 19.64 & 0.041 \\
\hline gcp95day2 & 46 & 450529.3 & 6574667 & 60.832 & 60.87 & 0.038 \\
\hline gcp65day2 & 47 & 449785.3 & 6582562 & 41.467 & 41.5 & 0.033 \\
\hline gcp-39day1 & 48 & 452676.5 & 6581857 & 45.989 & 46.02 & 0.031 \\
\hline gcp-41day1 & 49 & 452932.7 & 6581367 & 41.789 & 41.82 & 0.031 \\
\hline gcp-50day1 & 50 & 457478.5 & 6577277 & 19.35 & 19.38 & 0.03 \\
\hline gcp4day2 & 51 & 454884.6 & 6574200 & 447.132 & 447.16 & 0.028 \\
\hline gcp79day2 & 52 & 455089.5 & 6576426 & 19.524 & 19.55 & 0.026 \\
\hline gcp-51day1 & 53 & 457470.7 & 6577338 & 19.267 & 19.29 & 0.023 \\
\hline gcp53day2 & 54 & 446706.9 & 6584290 & 39.587 & 39.61 & 0.023 \\
\hline gcp67day2 & 55 & 451323.8 & 6580504 & 27.928 & 27.95 & 0.022 \\
\hline gcp51day2 & 56 & 446673.3 & 6584306 & 40.012 & 40.03 & 0.018 \\
\hline gcp68day2 & 57 & 451317.1 & 6580498 & 27.892 & 27.91 & 0.018 \\
\hline gcp82day2 & 58 & 455059.5 & 6576454 & 19.507 & 19.52 & 0.013 \\
\hline gcp99day2 & 59 & 450642.4 & 6574656 & 60.418 & 60.43 & 0.012 \\
\hline gcp9day2 & 60 & 453981.3 & 6574999 & 536.051 & 536.06 & 0.009 \\
\hline gcp80day2 & 61 & 455072.8 & 6576400 & 19.461 & 19.47 & 0.009 \\
\hline gcp152day2 & 62 & 452457.7 & 6582244 & 35.224 & 35.23 & 0.006 \\
\hline gcp96day2 & 63 & 450544.7 & 6574661 & 60.475 & 60.48 & 0.005 \\
\hline gcp55day2 & 64 & 448452.2 & 6583594 & 43.536 & 43.54 & 0.004 \\
\hline gcp98day2 & 65 & 450615.6 & 6574659 & 60.519 & 60.52 & 0.001 \\
\hline gcp-38day1 & 66 & 452620.3 & 6581799 & 34.52 & 34.52 & 0 \\
\hline gcp-35day1 & 67 & 452564.2 & 6581834 & 33.854 & 33.85 & -0.004 \\
\hline gcp66day2 & 68 & 449775.3 & 6582580 & 41.554 & 41.55 & -0.004 \\
\hline gcp12day2 & 69 & 450411.6 & 6579750 & 226.275 & 226.27 & -0.005 \\
\hline gcp-40day1 & 70 & 452732.8 & 6581885 & 52.048 & 52.04 & -0.008 \\
\hline hain320sday2 & 71 & 452453.8 & 6581862 & 33.32 & 33.31 & -0.01 \\
\hline hain320sday2 & 72 & 452453.8 & 6581862 & 33.32 & 33.31 & -0.01 \\
\hline gcp52day2 & 73 & 446697.3 & 6584307 & 39.591 & 39.58 & -0.011 \\
\hline gcp34day2 & 74 & 445631.4 & 6581599 & 65.762 & 65.75 & -0.012 \\
\hline gcp129day2 & 75 & 448341.2 & 6584957 & 38.735 & 38.72 & -0.015 \\
\hline gcp-26day1 & 76 & 452449 & 6581862 & 33.146 & 33.13 & -0.016 \\
\hline gcp-49day1 & 77 & 457472.8 & 6577189 & 19.718 & 19.7 & -0.018 \\
\hline gcp61day2 & 78 & 449816.6 & 6582593 & 41.051 & 41.03 & -0.021 \\
\hline gcp-21day1 & 79 & 468026.8 & 6569079 & 9.453 & 9.43 & -0.023 \\
\hline gcp64day2 & 80 & 449810.6 & 6582541 & 41.393 & 41.37 & -0.023 \\
\hline
\end{tabular}




\begin{tabular}{|c|c|c|c|c|c|c|}
\hline FID & & $\begin{array}{c}\text { Easting } \\
(\mathrm{m})\end{array}$ & $\begin{array}{l}\text { Northing } \\
\text { (m) }\end{array}$ & $\begin{array}{c}\text { Known Z } \\
(\mathrm{m})\end{array}$ & $\begin{array}{l}\text { Laser Z } \\
(\mathrm{m})\end{array}$ & $\mathrm{DZ}(\mathrm{m})$ \\
\hline gcp151day2 & 81 & 451713.4 & 6583319 & 33.756 & 33.73 & -0.026 \\
\hline gcp59day2 & 82 & 448457.6 & 6583542 & 44.007 & 43.98 & -0.027 \\
\hline gcp-33day1 & 83 & 452469.4 & 6581945 & 33.627 & 33.6 & -0.027 \\
\hline gcp127day2 & 84 & 448336.7 & 6584977 & 39.204 & 39.16 & -0.044 \\
\hline gcp57day2 & 85 & 448488.8 & 6583561 & 43.844 & 43.8 & -0.044 \\
\hline gcp56day2 & 86 & 448475 & 6583585 & 43.54 & 43.49 & -0.05 \\
\hline gcp-59day1 & 87 & 454804.7 & 6580555 & 26.104 & 26.05 & -0.054 \\
\hline gcp128day2 & 88 & 448371.8 & 6584980 & 40.469 & 40.41 & -0.059 \\
\hline gcp154day2 & 89 & 452442.5 & 6582320 & 35.383 & 35.32 & -0.063 \\
\hline gcp154day2 & 90 & 452442.5 & 6582320 & 35.383 & 35.32 & -0.063 \\
\hline gcp141day2 & 91 & 450934 & 6583978 & 36.73 & 36.66 & -0.07 \\
\hline gcp150day2 & 92 & 451691.1 & 6583342 & 33.915 & 33.84 & -0.075 \\
\hline gcp150day2 & 93 & 451691.1 & 6583342 & 33.915 & 33.84 & -0.075 \\
\hline gcp-43day1 & 94 & 457913.8 & 6576587 & 19.685 & 19.61 & -0.075 \\
\hline gcp156day2 & 95 & 452422.5 & 6582421 & 35.459 & 35.38 & -0.079 \\
\hline gcp156day2 & 96 & 452422.5 & 6582421 & 35.459 & 35.38 & -0.079 \\
\hline gcp160day2 & 97 & 452400.1 & 6582430 & 34.184 & 34.1 & -0.084 \\
\hline gcp160day2 & 98 & 452400.1 & 6582430 & 34.184 & 34.1 & -0.084 \\
\hline gcp1day2 & 99 & 452454.8 & 6581866 & 33.256 & 33.17 & -0.086 \\
\hline gcp-45day1 & 100 & 457854.5 & 6576668 & 19.149 & 19.06 & -0.089 \\
\hline gcp143day2 & 101 & 450914.6 & 6583978 & 36.55 & 36.46 & -0.09 \\
\hline gcp144day2 & 102 & 450909.2 & 6583966 & 36.67 & 36.58 & -0.09 \\
\hline gcp145day2 & 103 & 450930.1 & 6583940 & 36.251 & 36.16 & -0.091 \\
\hline gcp159day2 & 104 & 452394.3 & 6582459 & 34.221 & 34.13 & -0.091 \\
\hline gcp153day2 & 105 & 452450 & 6582282 & 35.385 & 35.29 & -0.095 \\
\hline gcp153day2 & 106 & 452450 & 6582282 & 35.385 & 35.29 & -0.095 \\
\hline gcp157day2 & 107 & 452409 & 6582491 & 35.448 & 35.33 & -0.118 \\
\hline gcp158day2 & 108 & 452390.9 & 6582489 & 34.626 & 34.5 & -0.126 \\
\hline gcp158day2 & 109 & 452390.9 & 6582489 & 34.626 & 34.5 & -0.126 \\
\hline gcp113day2 & 110 & 448033 & 6585947 & 86.398 & 86.27 & -0.128 \\
\hline gcp136day2 & 111 & 450951.7 & 6583935 & 35.68 & 35.54 & -0.14 \\
\hline gcp115day2 & 112 & 448062.2 & 6585924 & 87.487 & 87.34 & -0.147 \\
\hline gcp139day2 & 113 & 450970.4 & 6583947 & 36.485 & 36.33 & -0.155 \\
\hline gcp125day2 & 114 & 448262.8 & 6585794 & 94.283 & 94.12 & -0.163 \\
\hline gcp124day2 & 115 & 448268.1 & 6585798 & 94.367 & 94.18 & -0.187 \\
\hline gcp111day2 & 116 & 447998.6 & 6585978 & 84.958 & 84.77 & -0.188 \\
\hline gcp137day2 & 117 & 450973.8 & 6583926 & 36.194 & 36 & -0.194 \\
\hline gcp138day2 & 118 & 450986.1 & 6583935 & 36.451 & 36.25 & -0.201 \\
\hline gcp116day2 & 119 & 448080.6 & 6585911 & 88.144 & 87.94 & -0.204 \\
\hline gcp123day2 & 120 & 448251.7 & 6585808 & 93.823 & 93.6 & -0.223 \\
\hline gcp119day2 & 121 & 448137.3 & 6585875 & 90.043 & 89.81 & -0.233 \\
\hline
\end{tabular}




\begin{tabular}{|c|c|c|c|c|c|c|}
\hline FID & & $\begin{array}{c}\text { Easting } \\
(\mathbf{m})\end{array}$ & $\begin{array}{c}\text { Northing } \\
(\mathbf{m})\end{array}$ & $\begin{array}{c}\text { Known Z } \\
(\mathbf{m})\end{array}$ & $\begin{array}{c}\text { Laser Z } \\
(\mathbf{m})\end{array}$ & DZ (m) \\
\hline gcp117day2 & 122 & 448109.2 & 6585892 & 89.143 & removed & $*$ \\
\hline gcp120day2 & 123 & 448170.9 & 6585855 & 91.24 & removed & $*$ \\
\hline gcp121day2 & 124 & 448189.9 & 6585844 & 91.87 & removed & $*$ \\
\hline gcp118day2 & 125 & 448124.8 & 6585883 & 89.596 & removed & $*$ \\
\hline & & & & & & \\
\hline Average dz & & 0.000 & & & & \\
\hline Minimum dz & & -0.233 & & & & \\
\hline Maximum dz & & 0.178 & & & & \\
\hline $\begin{array}{c}\text { Mean magnitude of } \\
\text { deviation }\end{array}$ & & 0.077 & & & & \\
\hline \begin{tabular}{c} 
Root mean square \\
\hline $\begin{array}{c}\text { Standard deviation } \\
\text { (n=117) }\end{array}$
\end{tabular} & 0.096 & & & & \\
\hline
\end{tabular}

